TH4B-(SS2)-3

\title{
QUANTUM DOT STRUCTURES AND THEIR OPTICAL PROPERTIES OF A HIGH-INDIUM InGaN FILM
}

\author{
Shih-Wei Feng, En-Chiang Lin, Yung-Chen Cheng, Hsiang-Chen Wang, and C. C. Yang \\ Graduate Institute of Electro-Optical Engineering and Department of Electrical Engineering, \\ National Taiwan University, 1, Roosevelt Road, Sec. 4, Taipei, Taiwan, R.O.C. \\ (phone) 886-2-23657624 (fax) 886-2-2365263 (E-mail) ccy@cc.ee.ntu.edu.tw \\ Kung-Jen Ma \\ Department of Mechanical Engineering, Chung Hua University, Hsinchu, Taiwan, R.O.C. \\ Cheng-Hsing Shen and L. C. Chen \\ Center for Condensed Matter Sciences, National Taiwan University, Taipei, Taiwan, R.O.C. \\ K. H. Kim, J. Y. Lin and H. X. Jiang \\ Department of Physics, Kansas State University, Manhattan, Kansas, 66506-2601 USA
}

\begin{abstract}
Yellow luminescence from an InGaN film of high indium content shifted into blue emission upon thermal annealing. The shift was attributed to the quantum dot-like cluster size reduction through spinodal decomposition at thermal annealing.
\end{abstract}

We report the thermal annealing effects on the optical and material characteristics of a high-indium InGaN film with luminescence in the yellow range. It was observed that after thermal annealing, the PL peak shifted from the yellow band into the blue range. The spectral change was attributed to the reduction of cluster size upon thermal annealing. The sample used in this study was grown in a low-pressure MOCVD. A 1.2 $\mu \mathrm{m}$ GaN layer was deposited on a sapphire substrate with a $25 \mathrm{~nm} \mathrm{GaN}$ buffer layer. The GaN layer growth was followed by the deposition of an InGaN film with a thickness of $\sim 0.2 \mu \mathrm{m}$. The nominal indium content was estimated to be higher than $30 \%$. Thermal annealing was conducted with $800^{\circ} \mathrm{C}$ for $30 \mathrm{~min}$ in ambient nitrogen. Fig. 1 shows the PL and PLE spectra at $10 \mathrm{~K}$ of the as-grown and annealed samples. The PL spectral peak has shifted from the yellow band (around $2.2 \mathrm{eV}$ ) to the blue band (around $\cdot 2.75 \mathrm{eV}$ ) after thermal annealing. The blue emission contribution as a small side-lobe can also be observed in the PL spectrum of the as-grown sample. Fig. 2 shows the cathodo-luminescence spectra of the as-grown and annealed samples. In each sample, the $15 \mathrm{kV}$ and $3 \mathrm{kV}$ curves represent the results of using different electron acceleration voltages for deeper and shallower luminescence generations. In the as-grown sample, only the luminescence in the yellow band is observed in a shallow layer. However, with $15 \mathrm{kV}$ electrons, not only the strong luminescence of the GaN layer (around $2.4 \mathrm{eV}$ ) can be seen, but also a small peak of blue luminescence can be observed. Hence, it is expected that a certain microstructure existing deep in the InGaN film can emit photons in the blue range. After thermal annealing, in the shallow layer of InGaN ( $3 \mathrm{kV}$ probe), only the blue luminescence exists that is consistent with the PL measurement. With higher energy electron excitation, the blue luminescence is enhanced and the GaN emission peak appears. HRTEM images showed that the domain size in the annealed sample is smaller. Also, the domain density becomes higher after thermal annealing. Hence, we can assume that during thermal annealing, spinodal decomposition tends to break large clusters into smaller ones. In this process, the indium content in an InGaN cluster can be enhanced, leading to a smaller material band gap. However, the shrinkage of cluster size results in stronger quantum confinement and increases the effective band gap of the quantum dot. The quantum confinement effect may overcome the contradictory trend of indium content increase and result in a significant blue shift in PL spectrum.

Fig. 1

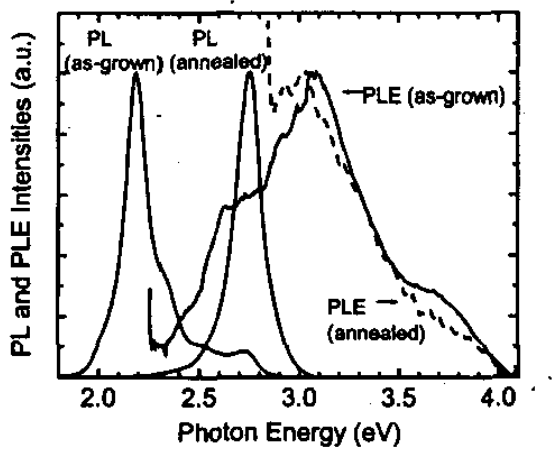

Fig. 2

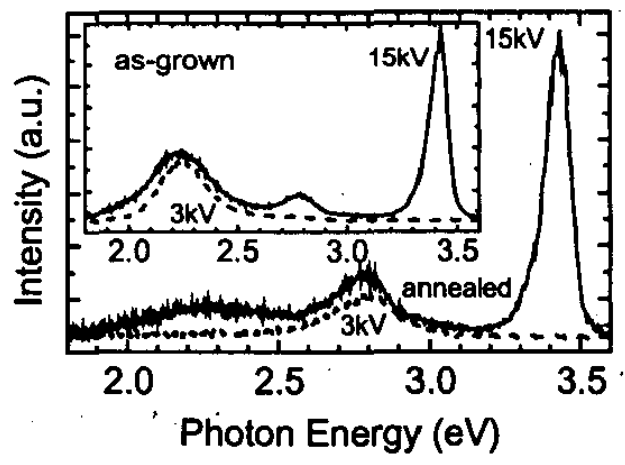

